\title{
ATUAÇÃO DA ENFERMAGEM NO GERENCIAMENTO DE RECURSOS MATERIAIS EM UNIDADES DE TERAPIA INTENSIVA (UTIS) ${ }^{1}$ \\ NURSING PERFORMANCE IN MATERIAL RESOURCES MANAGEMENT IN INTENSIVE CARE UNITS (ICUS)
}

\author{
LA PRÁCTICA DE LA ENFERMERÍA EN EL GERENCIAMENTO DE RECURSOS \\ MATERIALES EN UNIDADES DE TRATAMIENTO INTENSIVO ( UTIS)
}

\section{Cátia Romano ${ }^{2}$ Kátia Veiga ${ }^{3}$}

\begin{abstract}
RESUMO: Este estudo teve como objetivo identificar a atuação da enfermagem no gerenciamento de recursos materiais em hospitais gerais. Trata-se de um trabalho descritivo, de natureza quanti-qualitativa, realizado em quatro UTIs de hospitais gerais e de grande porte da cidade de Salvador-Ba. Observou-se que, das quatro unidades, em três há a presença do enfermeiro na comissão de seleção e compra de materiais. A participação da enfermagem no processo da previsão e controle de materiais é mínima, não havendo conseqüentemente uma avaliação do material que se está comprando, ocasionando a presença, muitas vezes, de materiais similares, ociosos e de má qualidade, comprometendo direta ou indiretamente a assistência prestada. Constatou-se que a atuação da enfermagem no gerenciamento de recursos materiais ficou restrita, apenas, à reposição destes nos serviços, sem, no entanto, inserir-se efetiva e eficazmente no processo de previsão, seleção, compra e controle desses insumos.
\end{abstract}

PALAVRAS-CHAVE: Atuação da enfermagem - Gerenciamento de materiais Recursos materiais.

ABSTRACT: This study had as a goal to identify the nursing practice in management of material resources in a general hospital. It is a descriptive work of quantiqualitative nature, performed in four ICU's (Intensive Care Units) among the largest general hospitals in Salvador-Bahia. It has been observed that (only) in three out of four Units, there was the presence of nurses in the commission of material purchase and selection. In addition, the participation of the nursing group in the process of material prevision and control is the lowest, there is lack of evaluation of purchased material; resulting, in turn, in the not occasional presence, of second line, useless and of low quality materials which directly or indirectly affect the provided care. It has been noticed that the nursing performance in material resources management became restrict, only for the reposition of these consumed materials, without, therefore, an effective and efficient insertion in the process of prevision, selection, purchasing and controlling.

KEYWORDS: Nursing practice - Management of material - Material resources.

\footnotetext{
${ }^{1}$ Trabalho apresentado no $49^{\circ}$ Congresso Brasileiro de Enfermagem - Belo Horizonte-MG, dezembro/1997.

${ }^{2}$ Professora Assistente da disciplina Administração e Planejamento dos Serviços de Saúde da EEUFBA e pesquisadora do Projeto Gerenciamento e Qualidade da Assistência em Unidade de Terapia Intensiva em Hospitais Gerais de Salvador- Ba./GEPASE.

${ }^{3}$ Professora Assistente da disciplina Administração e Planejamento dos Serviços de Saúde da EEUFBA; pesquisadora do Projeto Gerenciamento e Qualidade da Assisténcia em Unidade de Terapia Intensiva em Hospitais Gerais de Salvador-Ba./GEPASE; Enfermeira da Câmara Municipal de Salvador-BA.
} 
RESÚMEN: Este estudio tuvo como objetivo identificar la actuación de la enfermería en el gerenciamiento de recursos materiales en hospitales generales. Se trata de un trabajo descriptivo, de naturaleza cuanti-cualitativa, realizada en cuatro UTIs de hospitales generales y de grande tamaño, de la ciudad de Salvador -Ba. Fué observado que, de las cuatro unidades, en tres existe la presencia del enfermero en la comisión de selección y compra de materiales. La participación de la enfermería en el proceso de la previsión y control de materiales, es mínima, no habiendo, consecuentemente, una evaluación del material que se está comprando, ocasionando la presencia, muchas vezes, de materiales similares, ociosos y de mala calidad, comprometiendo directa o indirectamente la asistencia prestada. Quedó constatado que la actuación de la enfermería en el gerenciamento de recursos materiales se limitó, apenas, a la reposición de estos en el servicio, sin, apesar de esto,incluirse efectiva y efizcamente en el proceso de previsión, selección, compra y control de estos insumos.

PALABRAS CLAVE: Actuación de la enfermería - Gerenciamento de materiales Recursos materiales.

\section{INTRODUÇÃO}

A enfermagem, considerada contigente maior de trabalhadores nas Instituiçōes Hospitalares, segundo o Conselho Federal de Enfermagem (COFEn, 1985), tem nos recursos materiais insumos indispensáveis para a operacionalização do cuidar. Para tanto, tornou-se de sua responsabilidade a previsão, provisão, organização e controle em quantidade e qualidade suficiente para efetivar este processo. Segundo Maeda e Campadelli (1991), o serviço de Enfermagem utiliza a maior parte dos materiais hospitalares e, portanto, essa categoria tem a necessidade de opinar sobre os mesmos, tanto em relação à qualidade quanto à quantidade suficiente para suprir as necessidades de atendimento à sua clientela.

A prática, enquanto docentes na área de Administração Aplicada à Enfermagem, mostrou-nos que o enfermeiro tem pouca atuação no processo de gerência de materiais, restringindo sua atuação às atividade burocráticas, sem se inserir efetivamente no processo. Além do que percebemos também que são poucos os enfermeiros que conseguem fazer uma previsão de material eficaz para atender as necessidades dos setores, sem que haja excesso ou escassez de material.

Diante do exposto, pretendeu-se estudar a atuação da enfermagem no gerenciamento de recursos materiais em Instituiçőes Hospitalares na cidade de Salvador-BA, tendo como objetivos:

- Identificar e avaliar a atuação da enfermagem no gerenciamento de recursos materiais em UTIs Hospitais Gerais da cidade de Salvador-Ba. 
- Identificar a atuação da enfermagem no processo de aquisição de materiais em UTIs de Hospitais Gerais da cidade de Salvador - Ba.

- Avaliar a atuação da enfermagem nas funções de previsão, provisão, organização e controle de recursos materiais em UTIs de Hospitais Gerais da cidade de Salvador-Ba.

\section{METODOLOGIA}

Trata-se de um trabalho descritivo realizado em quatro UTIs de Hospitais Gerais da cidade de Salvador-Ba. As instituições em estudos têm como características comuns serem hospitais gerais e de grande porte. Segundo a entidade mantenedora, estas instituições se caracterizam da seguinte forma: uma Rede Federal, funcionando como Hospital-escola; outra filantrópica e duas da rede estadual, funcionando estas como referências enquanto unidade de emergência no município.

Utilizou-se como instrumento de coleta de dados a entrevista semi estruturada, com duração de aproximadamente 30 minutos, aplicada às enfermeiras responsáveis pelo Serviço de Enfermagem na UTIs, em fevereiro de 1997. Utilizou-se este tipo de entrevista por permitir maior liberdade nas respostas (Triviños, 1987).

\section{REVISÃO BIBLIOGRÁFICA}

Os recursos materiais são fundamentais ao funcionamento de uma organização, constituindo infra-estrutura indispensável para a produção de um bem ou serviço. A sua disponibilidade em tempo e local adequados favorece o desenvolvimento das atividades laborativas, reduzindo o custo e aumentando a produtividade.

Atualmente, vêm sendo administradas por setores especializados que centralizam todas as atividades relacionadas a materiais, sendo elas previsão, aquisição, transportes, recebimento, armazenamento, conservação, distribuição e controle (Castilho \& Leite, 1991).

Nas instituiçōes de saúde, cujo produto final é a prestação da assistência aos clientes através de ações terapêuticas que não podem sofrer interrupções, há a utilização de procedimentos e tratamentos complexos que exigem materiais cada vez mais modernos para sua execução.

Assim, nos hospitais utilizamos uma grande quantidade de materiais, que vão desde equipamentos sofisticados até produtos de limpeza, alimentos, roupas de camas, medicamentos, peças de reposição, materiais descartáveis, dentre outros. Os grandes hospitais manipulam cerca de 5000 itens, sendo os maiores usuários destes a farmácia, os serviços de nutrição e dietética, manutenção e reparo, lavanderia e enfermagem (Machline, 1983). 
$\mathrm{Na}$ prática, isso vem gerando uma série de problemas, visto que o mercado oferece uma grande diversidade de produtos, lançados quase que diariamente, 0 que dificulta a análise de desempenho e avaliação da qualidade, complicando a seleção dos mesmos, a qual deve contemplar critérios de desempenho, custos e facilidades de manuseio e manutenção. Essa demanda exige um grande empenho do usuário para manter-se atualizado acerca dessa evolução (Silva, Fernandes e Gonçalves, 1994).

A grande quantidade destes produtos dificulta incontestavelmente, a administração dos recursos materiais. Deste modo, a falta de padronização e de triagem, através de avaliações qualitativas e de custo - benefício, favorece o obsoletismo, a subutilização, extravios e danos, desperdicios, entre outros.

A padronização, em particular, proporciona resultados compensadores, tanto econômicos quanto técnicos, facilitando as operações de administração de materiais relacionados principalmente à previsão pela redução de itens, melhoria de organização e controle eficiente. Desse modo, a padronização é um processo de simplificação que contribui para a melhoria de qualidade dos produtos usados nos cuidados aos pacientes (Bittar, 1983).

Assim, o aumento na utilização de materiais e equipamentos de apoio à atividade médico - hospitalar tem melhorado indiscutivelmente a qualidade dos serviços prestados pelas instituições de saúde, o que vem alterando o processo diagnóstico - terapêutico, principalmente quanto à prevenção secundária e terciária, como se observa em unidades especializadas, em particular as UTIs (Unidades de Terapia Intensiva), segundo Dobson apud Madureira (1993).

Estas unidades valem-se de um aparato tecnológico em niveis de complexidade e variedade das mais diversas para auxiliar a monitorização e sustentação da vida, representando conseqüentemente um avanço no sentido da assistência ao cliente.

Ao mesmo tempo, tal avanço dificulta a proteção da equipe de saúde, e principalmente a de enfermagem, por se defrontar com materiais por vezes desconhecidos e cada vez mais sofisticados.

A equipe de saúde tem papel importante na administração dos recursos materiais, tendo envolvimento direto no processo aquisitivo por ser usuária desses materiais, pois o adequado provimento quanti-qualitativo garante sua atuação no contexto de segurança.

Surge, assim, a necessidade de escolha por um profissional qualificado, que conheça o materiais hospitalares de interesses da instituiçăo, contribuindo para a economia hospitalar através de compras de materiais adequados e menor desperdicio.

Neste contexto, a enfermagem, considerando o maior contingente de pessoal no hospital - 70, 4\% (COFEn - 1985), e que manipula esses materiais, tem no enfermeiro o profissional adequado para administrar este serviço, opinando e decidindo sobre os recursos mais apropriados à prestação de assistência qualificada segura aos usuários, pacientes e à própria equipe de saúde. 
As experiências da atuação do enfermeiro como gerente de recursos materiais têm-se mostrado altamente positivas, pois ele centraliza todas as atividades relacionadas a estes insumos, atuando como elo integrador entre as equipes de enfermagem e médica intermediando ainda, os contatos necessários com os serviços de compras, almoxarifado, entre outros (Silva, Fernandes, Gonçalves, 1994).

Nas unidades de enfermagem, a administraçäo desses recursos compreende 04 funções básicas (Castilho e Leite, 1991):

* Previsão e compras de materiais, entendido como o levantamento das necessidades da unidade, definindo tipo, quantidade e qualidade dos mesmos;

* Provisão, compreendido como a reposição dos materiais necessários ao desempenho de atividades de unidade;

* Organização, que consiste na disposição dos materiais da unidade, devendo atender a critérios de fácil acesso para uso e controle;

* Controle, relacionado à quantidade, qualidade, manutenção e proteção contra roubos e extravios na instituição.

Neste contexto, acreditamos que o enfermeiro, ao centralizar a gerência dessas atividades, será capaz de definir os materiais mais adequados à prestação de assistência com qualidade à clientela sob a sua responsabilidade, contribuindo para a racionalização dos recursos e melhor desempenho dos mesmos na organização.

\section{RESULTADOS}

\section{GRÁFICO 1 - ATUAÇÃO DA ENFERMAGEM NO PROCESSO DE AQUISIÇÃO DE MATERIAL.SALVADOR,1997.}

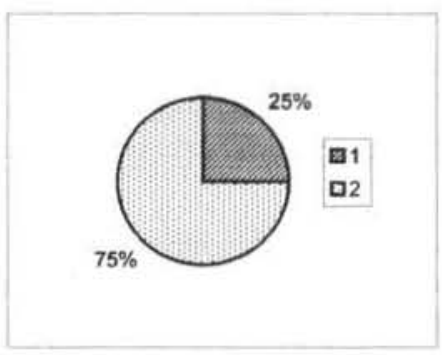
$1-25 \%=$ Atuação restrita nas comissర̋es de seleção e compra de material, de caráter formal.
$2-75 \%=$ Atuação direta nas comissőes de seleção e compra de material

Observamos neste estudo que $75 \%$ dos entrevistados informaram atuar diretamente nas comissões instituídas pelo hospital para selecionar e comprar todo material necessário ao seu funcionamento. Estas atuações foram identificadas nas instituições federal (hospital - escola), filantrópica e uma da rede estadual.

Nos hospitais-escola e no da rede estadual, o enfermeiro, apesar de inserirse na comissão de seleção e compra, emitindo pareceres técnicos e requisitando 
materiais de qualidade, nem sempre tem suas solicitaçōes atendidas pelo demais membros da comissão, pois predomina nestas instituições o critério do "menor preço unitário".

No hospital filantrópico, o enfermeiro participa ativamente do processo, sendo suas solicitações aceitas pela comissão de compra, por entender que este pedido é a melhor escolha para o desempenho da instituição.

Isto nos leva a crer que a realidade no âmbito hospitalar ainda não considera, nas decisőes de compra de material, a opinião da enfermagem, acarretando, na maioria das vezes, a aquisição de materiais de características adversas aos solicitados pelo serviço de enfermagem, implicando em produtos de qualidade inferior, ou ainda similares que não atendem às suas exigências, comprometendo, deste modo, a assistência global ao paciente.

A decisão relacionada à aquisição de material de qualidade comprovada é uma exigência atual que faz frente à evolução tecnológica vivenciada no direito de qualidade do consumidor, profissional ou cliente. Considerando que os materiais são necessários ao desenvolvimento das atividades assistenciais nos hospitais, os profissionais de saúde precisam dispor destes de forma que garantam uma atuação segura. Assim, torna-se necessário a inclusão dos profissionais de saúde na administração efetiva destes recursos (Silva, Fernandes e Gonçalves 1994).

Os $25 \%$ restantes informaram atuação restrita de caráter formal, compondo em número a comissão de seleção e compra de materiais na instituição, sem contudo ter poder de decisão. Alegam também acumular cargos, o que não lhes facultam tempo para desempenhar tão importante papel.

Em relação à previsão de material, observou-se que é feita basicamente utilizando a média de consumo das unidades do serviço de enfermagem, com pouca ou nenhuma preocupação com a real necessidade dos setores (estimativa de gastos). Isso gera, na maiorias das vezes, falta ou acúmulo de material, alguns dos quais essenciais à prestação do cuidado, interferindo, assim, na continuidade da assistência ao paciente. Outro fato citado é que os pedidos ao serem despachados pelos setores de almoxarifado e farmácia nem sempre correspondem à quantidade ou especificação técnica do enfermeiro. Assim, a estimativa de gasto dos materiais deixa a desejar, uma vez que não há cota estabelecida, acarretando as solicitações de compras extras, perdas e extravios dos mesmos.

Os entrevistados alegaram que os setores de materiais não têm estrutura para atender a solicitação dos serviços em tempo hábil, independente dos cronogramas estabelecidos para a distribuição nos diversos setores das instituições. Assim, a previsäo baseia-se no comportamento do material em determinado período de tempo com uma estimativa de gasto para o futuro, de sorte a atender todas as atividades a serem desenvolvidas nas unidades (Castilho e L eite, 1991).

Quanto ao controle, à exceção da instituição filantrópica, os entrevistados não fizeram referência à adoção de métodos específicos para tal. Em se tratando de UTI, sabe-se que os materiais e equipamento clínicos são essenciais para as ações terapêuticas e requerem pessoal qualificado para manuseá-los adequadamente e mantê-los prontos para uso. Neste contexto, a enfermagem desempenha importante papel no que se refere à supervisão e manutenção dos 
equipamentos a fim de assegurar uma assistência ininterrupta e segura à clientela e demais profissionais de saúde.

O controle de materiais contribui para a racionalização do trabalho, redução de gastos, extravios e danos, a estimativa de consumo médio de material nas diversas áreas dos hospitais e a garantia da integridade do material usado (Horr, 1989).

$\mathrm{Na}$ instituição filantrópica, está em estudo a implantação de um sistema informatizado de controle de material, com registro dos gastos individuais do paciente. À enfermeira caberá padronizar o material necessário ao desenvolvimento de suas atividades, confeccionando os denominados "Kits" por procedimento específico.

Alguns hospitais particulares começam a aderir à tendência mundial de automação de processos como item de referência para a qualidade total, personalizando o serviço ao paciente, utilizando processo informatizado de identificação e controle de materiais. Saem à frente do serviço público, que precisa enfrentar a burocracia para obter investimentos (Martinelli 1997).

Quanto à organização de materiais nas unidades, os entrevistados foram unânimes em dizer que utilizam a planta física dos diversos setores, armazenando estes materiais de maneira a facilitar o acesso aos funcionários, garantindo que não fiquem expostos à umidade e poeira dentre outros. Entretanto, não fizeram referência quanto às recomendações de armazenamento conservação e utilizaçäo de normas de operações dos equipamentos dos fabricantes, levando-nos a acreditar que os enfermeiros não entendem a importância destas normas para o uso adequado e seguro pelos pacientes e membros da equipe de saúde bem como a conservação dos mesmos, mantendo-os para uso.

Para facilitar a distribuição e o controle do material, é importante padronizar a sua localização nos vários setores. Esta medida, além de favorecer o processo como um todo, economiza tempo e energia do "Operador" (Horr, 1989).

Deste modo, acreditamos que, além da responsabilidade profissional do enfermeiro, tem que existir compromisso pessoal de atuar nestas decisões, compreendendo que a escolha adequada facilitará o desenvolvimento de suas atividades junto aos pacientes.

\section{CONSIDERAÇÕES FINAIS E SUGESTÕES}

O compromisso dos profissionais de saúde na administração de recursos materiais é imprescindivel para o uso racional e otimizado destes, que são importantes para o desenvolvimento da assistência segura e de qualidade à clientela sob a nossa responsabilidade bem como aos profissionais envolvidos no processo de assistir.

O enfermeiro demonstra ser o profissional tecnicamente preparado para assumir tal função, tanto por conhecer os materiais médico-hospitalares enquanto usuários, como por entender que a melhoria da qualidade da assistência prestada ao paciente depende, em parte, do material utilizado no hospital, fazendo-se necessária, então, a definição destes recursos por quem efetivamente os utiliza no dia-a-dia. 
Constatou-se com este estudo que, das quatro unidades, em três há a presença da enfermagem no processo de aquisição de materiais, com enfermeiros compondo a comissão de seleção e compra destes. A participação da enfermagem no processo de previsão e controle de materiais é minima, näo havendo, conseqüentemente, uma avaliação do material que se está utilizando, ocasionando a presença, muitas vezes, de materiais similares, ociosos e de má qualidade. A atuação da enfermagem na gerência de recursos materiais ficou restrita apenas à reposição destes recursos nos serviços sem, no entanto, inserir-se efetiva e eficazmente no processo de previsão, aquisição, controle e organização desses insumos.

Assim sendo, cabe ao enfermeiro : sistematizar as açöes de enfermagem no processo de aquisição; estabelecer requisitos mínimos essenciais para os materiais e elaborar fichas ou registros das características de qualidade dos materiais utilizados nos diversos serviços.

\section{REFERÊNCIAS BIBLIOGRÁFICAS}

1. BITTAR, Olimpio José Nogueira Viana.. Padronização de material. Rev. Paulista de Hospitais, Ano XXXI, vol. XXXI, n 5/6. Maio/Junho/1983.

2. CASTILHO, V.; LEITE, M.M.J.. A administração de recursos materiais na enfermagem In KURCGANT, Paulina (coord.) Administração em Enfermagem. São Paulo: E.P.U., 1991.

3. HORR, Lidvina. CIPRIANO, Zuimira M. \& RABELL, Ernesta S. Proposta de controle de material de consumo. R. Gaúcha de Enf. ; Porto Alegre, 10 (1):24-27.JAN. 1989.

4. MACHLINE, Claude. Recursos Materiais In GONÇALVES, Ernesto Lima (coord.) O hospital a visão administrativa contemporânea. 2 d. São Paulo: Pioneira, 1989.

5. MADUREIRA, Cátia M.. Romano. A enfermagem e os equipamentos clínicos: um estudo de caso em uma unidade de terapia intensiva. Dissertação de Mestrado. Escola de Enfermagem Anna Nery/UFRJ. 1993.

6. MAEDA, Sayuri Tanaka \& CAMPEDELLI, Maria Coeli. Escalpe e equipo de soro: opinião das enfermeiras quanto aos requisitos essenciais de qualidade. R. Bras. Enferm.; Brasilia, 44 (2/3): 124-135, abr/set. 1991.

7. MARTINELLI, Sílvia. Evolução saudável: aplicação do código de barras começa a ser prática comum nos principais laboratórios e hospitais do Pais. EAN Brasil - AC Revista - Automação Comercial. Ano IV, $n^{\circ} 44$. Junho/97.p.22-28.

8. SILVA, Sandra Honorato; FERNANDES, Rosa Aurea Quintella; GONÇALVES, Vera Lúcia Mira. A administração de recursos materiais: importância do enfoque de custos a responsabilidades dos profissionais de saúde. R. Bras. Enferm., Brasília, abr./Jun: 47(2) 1994. p. 160-64.

9. TRIVIÑOS, Augusto. Introdução à pesquisa em Ciências Sociais; a pesquisa qualitativa em educação. São Paulo: Atlas, 1987. 